\title{
Current-Carrying Zero Mode for the Nielsen-Olesen String
}

\author{
V.B. Svetovoy* \\ Department of Physics, Yaroslavl State University, \\ Sovetskaya 14, Yaroslavl 150000, Russia
}

\begin{abstract}
Zero modes of strings in the abelian Higgs model are analyzed. In spite of the fact that the gauge symmetry is not broken in the string center, the corresponding zero mode is shown to exist and to see it one has to analyze carefully the dependence on transverse coordinates for the excitations. The analysis of this kind is also important for the Witten model of superconducting string. Unusual properties of the zero modes connected with the broken gauge symmetry in the string background are investigated. One of the modes carries the current quite similar to that in the Witten model and gives back reaction to the string profile. It is claimed that the current in the string improves stability of the electroweak string.
\end{abstract}

PACS numbers: $11.27+\mathrm{d}, 98.80 . \mathrm{Cq}$

Keywords: cosmic string, zero mode, current, electroweak string

Cosmic strings are one dimensional topological defects which can appear in some spontaneously broken gauge theories during the phase transition [1]. The strings predicted by many Grand Unified Theories are important for cosmological applications [2]. They have been proposed as seeds for large scale structure formation [3] and as a means to reproduce temperature fluctuations in the cosmic microwave background [4.

*e-mail: vs@ics.ac.ru; tel. (0852) 1122 81; fax (0852) 116552 
Any string solution inevitably breaks some internal and space-time symmetries generating massless excitations of the string ("zero modes") connected with the broken transformations. In some cases these excitations are very important for the string dynamics. A well known example is the string superconductivity caused by the bosonic zero mode originated from the broken electromagnetic gauge symmetry inside the string core [5]. In the original Witten model the $\widetilde{U}(1) \times U(1)$ local symmetry is broken by the string solution, where the first factor gives rise to the Nielsen-Olesen vortex [6] and the second one (electromagnetic) becomes involved via interaction of the $\widetilde{U}(1)$ and $U(1)$ charged scalars. If the broken $U(1)$ symmetry generates a zero mode then why we should not expect a similar mode for the broken $\widetilde{U}(1)$ symmetry? This question was discussed in ref.[7] where a whole spectrum of the zero modes corresponding to the broken gauge symmetry and space-time rotations was described for the abelian Higgs model. However, possible physical manifestations of these modes have not been considered so far.

In this paper the physical role of the vortex zero modes in the abelian Higgs model will be analyzed. It will be shown that if the field equations are true for arbitrary distances, then these modes do not affect the physics. However, if there is a short-distance cutoff ( the Plank scale $M_{P}^{-1}$ is a natural candidate), one of them survives providing the hypercharge current along the string. We will see that this mode gives back reaction to the string forming fields and improves stability of the electroweak string.

To start with, one considers more complicated but familiar example that is the $\widetilde{U}(1) \times U(1)$ superconducting string [5]. Concerning the last $U(1)$ factor in this model there is a charged scalar field $\sigma$ which is condensed in the string core $\sigma=\bar{\sigma}(r)$ and an electromagnetic field $A_{\mu}$ vanishing for the string solution $A_{\mu}=\bar{A}_{\mu}=0$. Given a condensate, there is, in fact, a family of solutions for if $\sigma=\bar{\sigma}(r)$ is a solution of the field equations so is $\sigma=\bar{\sigma}(r) \exp (i \alpha)$. The phase is the Namby-Goldstone boson of the electromagnetic $U(1)$ symmetry, broken in the core of the string [2]. For the string along the $z$-axis the low-energy excitations have been written as [5]

$$
\sigma=\exp (i e \varphi(t, z)) \bar{\sigma}(r)
$$

where $\varphi(t, z)$ is arbitrary slowly varying function, $e$ is the electric charge of $\sigma$, and $r, \vartheta$ are the polar coordinates in the plain orthogonal to the $z$-axis. $\varphi(t, z)$ 
would be an exact zero mode if the $U(1)$ symmetry were not gauged, but for the local symmetry such an ansatz introduces nonzero current components $j_{0}$ and $j_{z}$ breaking the equations of motion for $A_{0}$ and $A_{z}$. It was assumed further that $A_{0, z}$ are the functions of $t$ and $z$ only whenever $\bar{\sigma} \neq 0$ and the problem was reduced to a 2-dimensional action for $\varphi(t, z)$ and $A_{0, z}(t, z)$. This is conventional logic introducing the current-carrying zero mode. It is obvious that this description is not absolutely correct since $A_{0, z}$ must depend on $r$ whenever $\bar{\sigma}(r)$ changes rapidly. Accurate analysis taking into account this dependence reveals unexpected properties of this zero mode.

Corrected ansatz [8] includes $r, \vartheta$ dependent phase $\varphi(t, z ; r, \vartheta)$ and excitation of the electromagnetic field. In the coordinate notations $x^{i}=(t, z)$ for $i=0,1$ and $y^{\alpha}=(r \cos \vartheta, r \sin \vartheta)$ for $\alpha=1,2$ it is

$$
\sigma=\exp (i e \varphi(x, y)) \bar{\sigma}(r), \quad A_{\mu}=\delta_{\mu}^{\alpha} \partial_{\alpha} \varphi(x, y),
$$

where the equations of motion for the function $\varphi(x, y)$ are

$$
\begin{gathered}
\partial_{\alpha}^{2} \varphi-2 e^{2} \bar{\sigma}^{2}(r) \varphi=0, \\
\partial_{i} \partial^{i} \varphi=0 .
\end{gathered}
$$

Gauge transforming by $\chi=-\varphi$ gives the alternative formulation

$$
\sigma=\bar{\sigma}(r), \quad A_{\mu}=-\delta_{\mu}^{i} \partial_{i} \varphi(x, y) .
$$

which shows that the zero modes are the excitations of the $i$-components (i.e. $t$ and $z$ ) of the gauge field.

Such a change in the description would be elaboration of a good qualitative description if not one circumstance: the solutions of eq.(3) are always singular at $r \rightarrow 0$ or $r \rightarrow \infty$. On this basis it was concluded [ 8 ] that the energy of the modes diverges and there must be a cutoff parameter for the theory to make sense.

A strict reader, probably, will propose here to exclude such excitations at all since they do not satisfy to the boundary conditions. However, they do. This question has been specially analyzed [7, 9] and it was shown that for some class of massless excitations against nontrivial classical backgrounds the boundary conditions are relaxed allowing some singular behavior. In contrast with [8] the energy of these modes was found to be finite. Usual excitations 
of the string are restricted at $r \rightarrow 0$ and vanish at $r \rightarrow \infty$. The modes under consideration extend the function space by generalized functions which are somewhat similar to the $\delta$-function: they allow singularities but their integral effect (energy) is finite.

To be more precise, one considers the abelian Higgs model where all the described effects also take place. The lagrangian of a system consisting of the $U(1)$ gauge field $B_{\mu}$ and the complex scalar field $\Phi$ is

$$
\mathcal{L}=-\frac{1}{4} F_{\mu \nu} F^{\mu \nu}+\left(D_{\mu} \Phi\right)^{*}\left(D_{\mu} \Phi\right)-\lambda\left(|\Phi|^{2}-\eta^{2} / 2\right)^{2}
$$

where

$$
F_{\mu \nu}=\partial_{\mu} B_{\nu}-\partial_{\nu} B_{\mu}, \quad D_{\mu} \Phi=\left(\partial_{\mu}-i g B_{\mu}\right) \Phi .
$$

One takes a Nielsen-Olesen string configuration [6] given by

$$
B_{i}=0, \quad B_{\alpha}=\frac{1}{r^{2}} \varepsilon_{\alpha \beta} y_{\beta} B(r), \quad \Phi=\Phi_{0}(r) e^{i \vartheta} .
$$

At the boundaries the functions $B(r)$ and $\Phi_{0}(r)$ behave as

$$
\begin{array}{r}
r \rightarrow 0: \quad B(r) \rightarrow 0, \quad \Phi_{0}(r) \rightarrow 0 ; \\
r \rightarrow \infty: \quad B(r) \rightarrow \frac{1}{g}, \quad \Phi_{0}(r) \rightarrow \frac{\eta}{\sqrt{2}} .
\end{array}
$$

The string properties are often treated on the basis of trapped false vacuum properties. The consequence of this approach is the conclusion that in the abelian Higgs model the gauge symmetry is unbroken in the string core and so there is no corresponding zero mode. However, such an approach is not quite correct. For example, on this way it would not be possible to predict the translational zero modes. Failure of the ansatz (1) is also the result of this approach. The string fields interpolate between false and true vacuua and definitely break the gauge symmetry so as the translations in $y_{\alpha}$ directions and the rotations between $x_{i}$ and $y_{\alpha}$ coordinates.

The translational zero modes are localized near the string core, regular and well known. They are not very interesting for our analysis. On the contrary, the modes connected with the broken gauge symmetry (gauge modes) and those arising as a result of the broken rotations (rotational modes) appear 
as very unusual subjects. A common method for these modes construction has been developed in ref. [9]. Let $V^{\mu}(x, y)$ and $\phi(x, y)$ be the excitations of the string fields $B^{\mu}$ and $\Phi$. The gauge can be chosen so that the rotational and gauge modes are contained only in $V^{i}$ [9] (see also (5)). Massless excitations $V^{i}(x, y)$ obey the equation

$$
\partial_{\alpha}^{2} V_{i}-2 g^{2} \Phi_{0}^{2}(r) V_{i}=0
$$

which, roughly speaking, is the condition of masslessness [. Gauge modes are the excitations solving eq.(91) and having the gradient form

$$
V^{i}(x, y)=\partial^{i} \varphi(x, y) .
$$

The rotational modes also have a special form

$$
V^{i}(x, y)=\delta g^{i \alpha}(x, y) B_{\alpha}(y),
$$

where $\delta g^{i \alpha}(x, y)$ is the metric variation under infinitesimal transformations of the coordinates. $V^{i}(x, y)$ one can expand in Fourier series

$$
V^{i}(x, y)=\sum_{m=0}^{\infty} V_{m \alpha}^{i}(x) e_{\alpha}^{(m)}(\vartheta) f_{m}(r),
$$

where $e_{\alpha}^{(m)}(\vartheta)=(\cos m \vartheta, \sin m \vartheta)$ and the functions $f_{m}(r)$ satisfy the equation

$$
f_{m}^{\prime \prime}+\frac{1}{r} f_{m}^{\prime}-\left(2 g^{2} \Phi_{0}^{2}(r)+\frac{m^{2}}{r^{2}}\right) f_{m}=0 .
$$

The solutions of this equation inevitably contain singularities at the origin or at infinity. Because of this special attention has to be paid to the boundary conditions for eq.(9). Typically boundary conditions for excitations come from the demand that the surface term in first variation of the action disappears. Any excitation $V^{i}(x, y)$ (massive or massless) is not restricted by this condition since the background components $B^{i}$ vanish. The boundary conditions on $V^{i}$ appear then from the demand of finiteness for the second variation of the action which is

\footnotetext{
${ }^{1}$ This equation has been deduced 9 analyzing the increase of the background fields under broken symmetry transformations.
} 


$$
S^{(2)}=\int d^{2} x d^{2} y\left[-\frac{1}{4}\left(\partial_{i} V_{j}-\partial_{j} V_{i}\right)^{2}+\frac{1}{2}\left(\partial_{\alpha} V_{i}\right)^{2}+g^{2}|\Phi|^{2} V_{i}^{2}\right] .
$$

For massless rotational or massive modes one finds the restriction

$$
\int d^{2} x d^{2} y \partial_{\alpha}\left(V_{i} \partial_{\alpha} V^{i}\right)=0
$$

which bounds massive modes at the boundaries in usual way and there is a nontrivial singular solution of eq.(15) for the rotational zero mode [7, 9]. The rotational mode needs special discussion that will be done elsewhere. Let us concentrate here on the gauge modes (10). For them the first term in (14) disappears and the rest ones give rise to the kinetic terms of the 2-dimensional fields $\varphi_{m \alpha}(x)$. In this case (15) can be realized restricting the $x$-dependence in usual way, $\int d^{2} x \partial_{i}\left(\varphi_{m \alpha} \partial^{i} \varphi_{m \alpha}\right)=0$, instead of bound the $y$-behavior of the modes.

Substituting the expansion (12) into the action (14) and integrating it over the $y_{\alpha}$ coordinates one finds a 2-dimensional lagrangian for the gauge modes

$$
\mathcal{L}^{(2)}=\sum_{m=0}^{\infty} \frac{Z_{m}}{2}\left(\partial^{i} \varphi_{\alpha m}(x)\right)^{2},
$$

where $V_{m \alpha}^{i}(x)=\partial^{i} \varphi_{\alpha m}(x)$ and the coefficients $Z_{m}$ are

$$
Z_{m}=\pi\left(1+\delta_{0 m}\right) \int d r \frac{d}{d r}\left(r f_{m} f_{m}^{\prime}\right) .
$$

This is what was expected for massless excitations. Although the integral in (17) diverges, the action is not infinite because one has to normalize $\varphi_{\alpha m}(x)$. The fields $\varphi_{\alpha m}(x)$ will be defined as 2-dimensional fields if the coefficients $Z_{m}=1$. This is usual normalization condition for eigenfunctions which has to be imposed on any kind of excitations. Since the functions $f_{m}(r)$ are singular at $r \rightarrow 0$, the normalization condition has to be defined with the help of a cutoff parameter $r_{0} \rightarrow 0$ :

$$
\pi\left(1+\delta_{0 m}\right) \int_{r_{0}}^{\infty} d r \frac{d}{d r}\left(r f_{m} f_{m}^{\prime}\right)=1
$$


In contrast with the case of regular eigenfunctions (18) can be considered as a boundary condition which together with eq.(13) completely defines the functions $f_{m}(r)$. On the other hand the condition (18) introduces generalized functions. Really, the set of solutions of eq. (13) normalized according (18) for $r_{0} \leq r \ll R$ has the form

$$
\begin{gathered}
f_{0}(r)=\frac{\ln (R / r)}{\sqrt{2 \pi \ln \left(R / r_{0}\right)}}, \\
f_{m}(r)=\frac{1}{\sqrt{\pi m}}\left(\frac{r_{0}}{r}\right)^{m}, \quad m>0,
\end{gathered}
$$

where $R \sim g \eta$ is the string radius. Similar to the $\delta$-function this functions vanish at any finite $r$ and take some value at $r=r_{0} \rightarrow 0$. The integral condition for the $\delta$-function is replaced by the normalization condition (18). So, our zero modes live strictly in the string center and obviously carry finite energy. The conclusion of ref. 88 that the energy of similar modes diverges was made because the normalization condition was not imposed and the energy was defined up to an arbitrary factor.

The $m=0$ mode induces charge per unit length and current of $U(1)$ charges along the string. The current density is

$$
j_{i}=-2 g^{2} \partial_{i} \varphi(x, y) \Phi_{0}^{2}(r) .
$$

Integrating $j_{1}$ over the string section with the help of (12) and (13) one finds for the current along the string

$$
J=\int j_{1} d^{2} y=\partial_{1} \varphi_{0}(x) \sqrt{\frac{2 \pi}{\ln \left(R / r_{0}\right)}},
$$

where the function $\varphi_{0}(x)$ so as any $\varphi_{m \alpha}(x)$ obeys the 2-dimensional wave equation. Note that the integral in (21) is defined by the asymptotics of $f_{0}(r)$ but not the condensate distribution $\Phi_{0}(r)$. The same result (21) will be found for the electric current in the Witten model in spite of difference in the condensates distribution. It differs from the expression found in [5] by the numerical factor but the qualitative result does not change. The reason for this difference, as was explained before, is taking into account $r$-dependence for the zero mode. 
In the limit $r_{0} \rightarrow 0$ the current $J$ goes to zero. However, in physical reality we cannot believe in our equations for arbitrary small distances. A natural candidate for the cutoff parameter $r_{0}$ is the Plank scale $M_{P}^{-1}$. With such a cutoff the current remains practically unsuppressed even if the string was formed at the electroweak transition when $R \sim M_{W}^{-1}$. The gauge modes are concentrated near the string center because of their singular nature. Indeed, all the $m>0$ modes cannot influence low energy physics since they vanish rapidly at a distance from the center large in comparison with $M_{P}^{-1}$. In contrast with that the $m=0$ mode decreases logarithmically and survives at large distances. Existence of the current itself is the direct result of the short distance behavior.

One suppose $\varphi_{0}(t, z)$ varies linearly with time and position

$$
\varphi_{0}(t, z)=b_{0} t-b_{1} z .
$$

In this case the string is described by the time and position independent fields

$$
B_{i}=b_{i} f_{0}(r), \quad B_{\alpha}=\frac{1}{r^{2}} \varepsilon_{\alpha \beta} y_{\beta} B(r), \quad \Phi=\Phi_{0}(r) e^{i \vartheta},
$$

and can be considered as a ground state with charge and current. The zero mode gives back reaction to the string profile because the equation for $\Phi_{0}(r)$ includes an additional term $g^{2}\left(b_{0}^{2}-b_{1}^{2}\right) f_{0}^{2} \Phi_{0}$.

The Nielsen-Olesen vortex solution can be embedded into the $S U(2)_{L} \times$ $U(1)_{Y}$ electroweak theory [10] and it is known as electroweak string. However, there are no topological arguments for stability of this string and it happens so that dynamical stability is reached outside of the physical region of parameters [11]. Perturbation spectrum for the electroweak string in presence of charge and current is modified. The problem will be analyzed elsewhere but to see the tendency one makes a few comments on the case $\sin ^{2} \theta_{W}=1$ (semilocal string [12]). The string profile is described by eq.(23), where $\Phi$ is the down component of the Higgs doublet and one has to replace $B_{\mu} \rightarrow Z_{\mu}$ and $g \rightarrow q$. One can show that in our case the stability analysis can be reduced to an analysis of the excitations in the upper component of the Higgs doublet $\phi$. Since the background fields do not depend on $t$ and $z$, $\phi$ can be sought in the form

$$
\phi(x, y)=e^{i \omega t} e^{-i k z} \phi(r, \vartheta)
$$


Expanding $\phi(r, \vartheta)$ in angular momentum states $\phi_{m}(r) e^{i m \vartheta}$ one comes to the eigenvalue problem for $\omega$

$$
\begin{gathered}
\phi_{m}^{\prime \prime}+\frac{1}{r} \phi_{m}^{\prime}+\left[\left(\omega+q b_{0} f_{0}\right)^{2}-\left(k+q b_{1} f_{0}\right)^{2}\right] \phi_{m}- \\
{\left[\frac{(m-q B)^{2}}{r^{2}}+2 \lambda\left(|\Phi|^{2}-\eta^{2} / 2\right)\right] \phi_{m}=0}
\end{gathered}
$$

One can immediately conclude that the current $\left(b_{1} \neq 0\right)$ improves string stability but the charge on the string $\left(b_{0} \neq 0\right)$ acts in the opposite direction. Therefore, it is expected that stability of the current-carrying electroweak strings will be improved.

In conclusion, it was shown that the current-carrying zero mode exists in the abelian Higgs model with the Nielsen-Olesen string solution. The argument that the gauge symmetry is unbroken inside of the string core does not work because the zero mode is connected rather with the transition between vacuua inside and outside of the string. The mode has logarithmic singularity in the string center, but it is normalizable in the sense of generalized

functions. The current along the string disappears as $1 / \sqrt{\ln \left(R / r_{0}\right)}$ when the short distance cutoff $r_{0}$ goes to zero. The physical cutoff parameter $r_{0} \sim M_{P}^{-1}$ leaves the current practically unsuppressed. It was claimed that the current in the string improves stability of the electroweak string.

\section{Note added}

After the submission of this paper author received a preprint by Kibble et al [13] in which a nonabelian current-carrying string configuration similar to (23) is considered. They investigated the gauge zero mode which has logarithmic singularity at infinity and instead of the normalization condition for the mode have connected behavior at large distances with the current in the string.

\section{References}

[1] T.W.B. Kibble, J. Math. A9 (1976) 1387; Phys. Rep. 67 (1980) 183.

[2] M.B. Hindmarsh and T.W.B. Kibble, Rep. Prog. Phys. 58 (1995) 477. 
[3] A. Vilenkin, Phys.Rep. 121 (1985) 263.

[4] B. Allen and E.P.S. Shellard, Phys. Rev. Lett. 64 (1990) 119.

[5] E. Witten, Nucl. Phys. B249 (1985) 557.

[6] H.B. Nielsen and P. Olesen, Nucl. Phys. B61 (1973) 45.

[7] N.G. Khariton and V.B. Svetovoy, Phys. Lett. B286 (1992) 53.

[8] M.G. Alford, K. Benson, S. Coleman, J. March-Russel and F. Wilczek, Phys. Rev. Lett. 64 (1990) 1632; Nucl. Phys. B349 (1991) 414.

[9] N.G. Khariton and V.B. Svetovoy, Phys. Rev. D49 (1994) 2982.

[10] T. Vachaspati, Phys. Rev. Lett. 68 (1992) 1977.

[11] M. James, L. Perivolaropoulos and T. Vachaspati, Nucl. Phys. B395 (1993) 534 .

[12] T. Vachaspati and A. Achucarro, Phys. Rev. D44 (1991) 3067.

[13] T.W.B. Kibble, G. Lozano and A.J. Yates, preprint Imperial/TP/9697/14, hep-ph/9701240. 